\title{
Fatores de risco para doenças cardiovasculares em crianças e adolescentes com sobrepeso e obesidade
}

\author{
Risk factors for cardiovascular diseases in children and adolescents with \\ overweight and obesity
}

\section{Adriana Masiero Kühla, Tatiana Ferreirab ${ }^{b}$, Angélica Rocha de Freitas Melhemc, Sandra Aires Ferreirad,} Timothy Gustavo Cavazzotto ${ }^{e}$, Marcos Roberto Queiroga ${ }^{f}$

a Nutricionista. Mestre em Nutrição e Dietética pela Universidade Federal de Santa Catarina. Docente da Universidade Estadual do Centro-Oeste (Unicentro).

${ }^{b}$ Nutricionista. Especialista em Nutrição Clínica e Alimentos Funcionais pela Universidade Estadual de Londrina (UEL). Residente na Universidade Federal do Mato Grosso do Sul.

c Nutricionista. Doutora em Ciências (Gastroenterologia) pela Universidade Federal de São Paulo (UNIFESP). Docente da Unicentro.

dEducadora Física. Mestre em Educação Física pela UNESP-RC e Doutoranda em Educação Física pela UEL.

e Educador Físico. Mestre e Doutorando em Educação Física pela UEL.

'Educador Físico. Doutor em Biodinâmica da Motricidade Humana pela Universidade Estadual Paulista (UNESP-RC). Docente da Unicentro.

Financiamento: Secretaria de Estado da Ciência, Tecnologia e Ensino Superior (SETI) do Paraná. Programa de Extensão Universidade sem Fronteiras (USF), Subprograma Apoio à Saúde (Edital 02/2011 e convenio 6/2012/SETI/UGF).

RESUMO

Objetivo: Descrever a prevalência de fatores de risco para doenças cardiovasculares de acordo com o sexo e o percentual de gordura $(\% \mathrm{G})$ em crianças e adolescentes com sobrepeso e obesidade.

Materiais e Métodos: Participaram do estudo 53 crianças e adolescentes (27 meninos e 26 meninas de 6 a 16 anos) com sobrepeso e obesidade que foram submetidos a medidas de massa corporal $(\mathrm{MC})$, estatura e circunferência da cintura (CC), bioimpedância (\%G) e coleta sanguínea para determinação das concentrações de glicose e perfil lipídico (TG, CT, HDL-C, LDL-C). O índice de massa corporal foi calculado (IMC=MC kg/estatura m²), IMC z-score foi empregado para classificação do estado nutricional e o índice $\operatorname{HOMA-RI}(>2,5)$ para resistência à insulina (RI).

Resultados: As meninas demonstraram, para mesma idade, maior MC e CC do que os meninos. Quando classificados de acordo com o \%G, observou-se que o grupo com valores determinados em alto (acima de $25 \%$ meninos e $30 \%$ meninas) apresentaram níveis de CT e LDL-C superior aos participantes com valores moderadamente altos (entre 20$25 \%$ meninos e $25-30 \%$ meninas). Grande parte dos participantes demonstram classificação de risco cardiovascular alterados para \%G (77,5\%), CC (64,2\%), HDL-C (67,9\%), CT (45,3\%), LDL-C (24,5\%), TG (41,5\%) e RI (49,5\%).

Conclusão: A prevalência de fatores de risco para doenças cardiovasculares é elevada neste grupo de crianças e adolescente com sobrepeso e obesidade e medidas de prevenção primária deverão ser implantadas.

Palavras-chave: crianças; adolescentes; dislipidemia; obesidade; fatores de risco; doenças cardiovasculares.

ABSTRACT

Objective: To describe the prevalence of risk factors for cardiovascular diseases according to gender and percentage body fat (\%BF) in overweight and obese children and adolescents.

Materials and Methods: Fifty-three children and adolescents (27 boys and 26 girls aged 6 to 16 years) who were overweight and obese underwent body mass (BM), height and waist circumference (WC) measurements, bioimpedance (\%BF) and blood collection for determination of glucose, and lipid profile (TG, CT, HDL-C, LDL-C). The body mass index was calculated (BMI=BM $\left.\mathrm{kg} / \mathrm{height} \mathrm{m}^{2}\right)$ and BMI Z-score was used to classify the nutritional status and the HOMA-IR index (> 2.5) for insulin resistance (IR).

Results: The girls showed, for the same age, higher BM and WC than boys. When classified according to the \%BF, it was observed that the group with values determined as high (above $25 \%$ boys and $30 \%$ girls) presented higher levels of CT and LDL-C than participants with moderately high values (between $20-25 \%$ boys and $25-30 \%$ girls). Most participants demonstrate altered cardiovascular risk $\% B F(77.5 \%)$, WC (64.2\%), HDL-C (67.9\%), CT (45.3\%), LDL-C (24.5\%), TG (41.5\%) and IR (49.5\%).

Conclusion: The prevalence of risk factors for cardiovascular diseases is high in this group of overweight and obese children and adolescents and primary prevention measures should be implemented. 


\section{INTRODUÇÃO}

A prevalência da obesidade no Brasil e no mundo vem progredindo acentuadamente sem distinção de classe social, idade e sexo constituindo um importante desvio nutricional que merece a atenção dos profissionais de saúde. Ela é caracterizada como uma doença crônica não transmissível (DCNT) de origem multifatorial com a interação de fatores genéticos, fisiológicos, ambientais e psicológicos que estão envolvidos na sua gênese, e acarretam no acúmulo excessivo de gordura corporal ${ }^{1,2}$.

No Brasil, entre outros, foram realizados levantamentos sobre a prevalência de excesso de peso e obesidade por meio do VIGITEL ${ }^{3}$ (Vigilância de Fatores de Risco e Proteção para Doenças Crônicas por Inquérito Telefônico) e da POF $^{4}$ (Pesquisa de Orçamentos Familiares). Comparando a prevalência entre os períodos de 2007 a 2009 observouse aumento de 43,4 para $46,6 \%$ no excesso de peso e de 12,7 para $13,9 \%$ na obesidade 3 . Por sua vez, no levantamento relizado pela POF, que compreendeu o período de 2008-2009, o excesso de peso foi observado em 33,5\% das crianças entre cinco a nove anos enquanto, $16,6 \%$ e $11,8 \%$ dos meninos e meninas, respectivamente, apresentavam obesidade $^{4}$.

A presença da obesidade é uma condição de risco para várias doenças e tem sido associada a alterações metabólicas, as quais contribuem para o aumento do risco de desenvolvimento de doenças cardiovasculares (DVC) ${ }^{1}$. A literatura apresenta fortes evidências no sentido de que as DCV, manifestadas na idade adulta, resultam de complexa interação entre uma variedade de fatores de risco que podem ter origem na infância e na adolescência. Estudos em crianças indicam que o processo da aterosclerose se inicia em idade precoce, ressaltado que o risco aumenta quando se trata de adolescentes obesos ${ }^{5,6}$.

A investigação dos fatores de risco vem sendo importante pela possibilidade de identificar novos caminhos para a prevenção e o tratamento das DCV, diminuindo assim a mortalidade e morbidade. Os fatores atualmente reconhecidos nesse meio são pressão arterial elevada, excesso de peso, deposição central de gordura corporal, dislipidemias, alterações do metabolismo da glicose, resistência à insulina e atividade física insuficiente ${ }^{7,8}$. Esses fatores tendem a se agregar e, frequentemente, são encontrados em associação no mesmo indivíduo, aumentando a probabilidade das DCV ${ }^{8}$.

Frente a esse panorama, é evidente a necessidade de um maior esclarecimento sobre o impacto da obesidade na saúde de crianças e adolescentes, o que poderia auxiliar na redução de morbidades e mortalidades no futuro, o que atualmente acarreta em um elevado custo econômico para a saúde pública. Diante disso, o objetivo foi descrever a prevalência de fatores de risco para doenças cardiovasculares de acordo com o sexo e o \%G em crianças e adolescentes com sobrepeso e obesidade.

\section{MATERIAIS E MÉTODOS}

Estudo transversal realizado com crianças e adolescentes de ambos os sexos com idade entre 6 e 16 anos, inseridos em um projeto de extensão intitulado "Programa de controle e tratamento da obesidade em crianças e adolescentes no município de Guarapuava-PR". O projeto obteve financiamento por meio do Edital 02/2011 do Programa de Extensão Universidade sem Fronteiras, Sub-Programa Apoio à Saúde, da Secretaria de Estado da Ciência, Tecnologia e Ensino Superior (USF/SETI) do Paraná e foi realizado no ano de 2104 (janeiro a dezembro). O estudo foi aprovado pelo Comitê de Ética em Pesquisa (parecer no 297.649/2013) da Universidade Estadual do Centro-Oeste (UNICENTRO) e todos os responsáveis pelos participantes assinaram o Termo de Consentimento Livre e Esclarecido (TCLE).

Houve divulgação em jornais, rádio e televisão e as escolas estaduais e particulares do município foram visitadas com anuência do Núcleo Regional de Educação. Após divulgação, 120 crianças e adolescentes, por meio de seus pais/responsáveis, efetivaram inscrição no projeto, das quais 101 inscritos passaram pela triagem inicial com avaliação antropométrica, alimentar, motora e psicológica. Essa perda inicial da amostra ocorreu por erro no preenchimento da inscrição e pelo não comparecimento dos participantes na data agendada para avaliação. Posteriormente os participantes foram encaminhados para a realização de exames bioquímicos em um laboratório de análises clínicas conveniado ao projeto, dos quais 65 indivíduos compareceram para a coleta de sangue. Outra etapa da avaliação ocorreu na Clínica Escola de Nutrição da Universidade Estadual do Centro-Oeste, onde foi realizado o teste de bioimpedância elétrica tetrapolar para quantificação do percentual de gordura corporal (\%G), na qual compareceram 58 participantes.

Os critérios de inclusão para o estudo foram os participantes terem completado todas as fases de avaliação, que incluiu a avaliação inicial, as medidas antropométricas, os exames bioquímicos e a bioimpedância. Por sua vez, o critério de exclusão foi o desejo de não fazer parte do estudo, caso que não foi revelado por nenhum responsável/ participante. Ao final de todo o processo de coleta de informações, foram incluídos nas análises do presente estudo 53 crianças e adolescentes com sobrepeso e obesidade.

Foram definidos como fatores de risco cardiovascular valores alterados para perfil lipídico (HDL-C, LDL-C, TG 
e CT) e glicêmico, excesso de peso, \%G e CC, bem como a resistência à insulina, obtida por meio do índice HOMA-RI (Homeostasis Model Assessment-Insulin Resistance).

As medidas antropométricas foram aferidas seguindo as recomendações de Lohman et al. ${ }^{9}$. A mensuração da massa corporal $(\mathrm{MC})$ foi realizada em uma balança da marca Welmy ${ }^{\circledR}$, com precisão de $0,1 \mathrm{~kg}$ e a medida da estatura foi obtida em um estadiômetro de madeira com precisão de $0,1 \mathrm{~cm}$. Para ambas as mensurações, os indivíduos encontravam-se com roupas leves e descalços. O índice de massa corporal $\left(\mathrm{IMC}=\mathrm{MC} \mathrm{kg} / \mathrm{estatura} \mathrm{m}^{2}\right)$ foi utilizado para a classificação do estado nutricional. Para os pontos de corte do IMC foram empregados os padrões da WHO z-score ${ }^{10}$.

A circunferência da cintura (CC) foi obtida no ponto médio entre as últimas costelas e a crista-ilíaca, por meio de uma fita métrica flexível, porém não elástica com precisão de $1 \mathrm{~mm}$, com o indivíduo em posição anatômica. Para classificação das medidas de CC em valores normais ou aumentados, foram utilizadas às recomendações de Freedman et al. ${ }^{11}$.

O percentual de gordura (\%G) foi estimado por meio de Bioimpedância elétrica (Maltron Body Fat Analyser BF-906 ${ }^{\circledR}$ England). Os participantes do estudo foram orientados a seguir alguns procedimentos prévios, sem os quais os resultados poderiam ser comprometidos: jejum absoluto de 12 horas; não realizar exercícios físicos extenuantes nas 12 horas anteriores ao teste; não ingerir álcool 48 horas antes da realização do teste; não ingerir medicamentos que influenciem o equilíbrio hidroeletrolítico (diuréticos, corticosteroides, entre outros) a menos de 7 dias do teste e urinar pelo menos 30 minutos antes da realização do teste. Os participantes foram classificação segundo recomendações de Lohman et al. ${ }^{12}$ que divide os valores de gordura corporal relativa em: Baixo $<10,0$ meninos; $<15,0$ meninas; Ótimo $\geq 10,0$ e $\leq 20,0$ meninos; $\geq 15,0$ e $\leq 25,0$ meninas; Moderadamente alto $>20,0$ e $<25,0$ meninos; $>25,0$ e $<30,0$ meninas e; Alto $\geq 25,0$ meninos; $\geq 30,0$ meninas. Em nosso estudo, os participantes apresentaram valores correspondentes as classificações Moderadamente alto e Alto.

As crianças e adolescentes, acompanhados de seus responsáveis, compareceram a um laboratório de análises clínicas do município para coleta sanguínea em condição de jejum noturno (10 a 12 horas). A coleta foi realizada mediante punção da veia antecubital utilizando o sistema de coleta a vácuo (Vacutainer ${ }^{\mathrm{TM}}$ Becton Dickinson Company, Plymouth, Reino Unido), utilizando tubos de $4,0 \mathrm{~mL}$ com anticoagulante (fluoreto associado ao EDTA $1 \mathrm{mg} / \mathrm{mL}$ sangue) e tubos de $3,5 \mathrm{~mL}$ com heparina. O plasma foi utilizado para determinação da concentração de Colesterol Total (CT),
Triglicerídeos (TG), High DensityLipoproteins-Colesterol (HDL-c), da glicose e insulina.

As determinações plasmáticas dos TG, CT, HDL-C e glicose foram realizadas pelo método colorimétrico enzimático. A insulina plasmática foi determinada pelo método ELISA, com a utilização de kits ultrassensíveis e coeficientes de variação intraensaio e interensaio de 5,96 $\pm 1,17 \mu \mathrm{U} / \mathrm{mL}$ e 10,3 $\pm 0,9 \mu \mathrm{U} / \mathrm{mL}$, respectivamente. A fração Low Density Lipoproteins-Colesterol (LDL-C) foi estimado por meio da fórmula de Friedewald: $\mathrm{LDL}-\mathrm{C}=\mathrm{CT}-\mathrm{HDL}-(\mathrm{TG} / 5)^{13}$.

Os parâmetros do metabolismo de glicose e lipídio foram classificados de acordo com os seguintes pontos de corte: Glicose: <100,0 mg/dL; CT: <150,0 mg/dL; HDL-C: $>45,0 \mathrm{mg} / \mathrm{dL}$; LDL-C: $<100,0 \mathrm{mg} / \mathrm{dL}$; TG: $<100,0 \mathrm{mg} / \mathrm{dL}^{14}$. $\mathrm{O}$ método utilizado para a avaliação da resistência à insulina foi o índice HOMA-RI (Homeostasis Model AssessmentInsulin Resistance), calculado a partir da fórmula ${ }^{15}$ : HOMA$\mathrm{RI}=$ insulinemia de jejum ( $\mathrm{mU} / \mathrm{L}) \times$ glicemia de jejum $(\mathrm{mmol} / \mathrm{L}) / 22,5$. A RI foi definida a partir dos valores de HOMA acima de 2,5 unidades $^{16}$.

Para a análise estatística os participantes foram divididos de acordo com o sexo e o \%G. Inicialmente foi verificada a distribuição e homogeneidade dos dados empregando os testes de Shapiro-Wilk e Levene, respectivamente. Os resultados foram apresentados em valores de média e $( \pm)$ desvio padrão ou frequência absoluta e relativa quando apropriado. O teste t de Student (grupos independentes) foi empregado para comparação entre os grupos. Os dados foram analisados com auxílio do pacote estatístico SPSS 20.0 e foi adotado nível de significância de $p<0,05$.

\section{RESULTADOS}

Após todos os procedimentos de coleta de dados (triagem inicial, avaliação antropométrica, alimentar, motora e psicológica), 53 crianças e adolescente, dos quais 26 eram meninas $(49,1 \%)$ e 27 eram meninos $(50,9 \%)$, com média de idade de 11,1 $\pm 2,6$ anos, participaram do estudo (Tabela 1 e Figura 1). Em relação ao estado nutricional todos os participantes apresentaram massa corporal acima do desejado, dos quais 18,9\% foram classificados com sobrepeso $(n=10), 60,4 \%$ com obesidade $(n=32)$ e $20,8 \%$ com obesidade severa $(n=11)$ (Tabela 3).

A descrição dos valores médios das crianças e adolescentes com sobrepeso e obesidade divididos em meninos e meninas e amostra total referente as características antropométricas, laboratoriais e da composição corporal encontram-se na Tabela 1. As meninas demonstraram significativamente maior MC e CC do que os meninos. Para outras variáveis, inclusive a idade, meninos e meninas com sobrepeso e obesidade, não apresentaram diferenças. 
Tabela 1. Caracterização da amostra de acordo com o sexo, variáveis antropométricas, laboratoriais e de composição corporal.

\begin{tabular}{lcccc}
\hline Variáveis & $\begin{array}{c}\text { Todos } \\
(\mathbf{n = 5 3})\end{array}$ & $\begin{array}{c}\text { Meninos } \\
(\mathbf{n = 2 6})\end{array}$ & $\begin{array}{c}\text { Meninas } \\
(\mathbf{n = 2 7})\end{array}$ & $\boldsymbol{p}^{*}$ \\
\hline Idade (anos) & $11,1 \pm 2,6$ & $10,9 \pm 2,6$ & $11,2 \pm 2,8$ & 0,731 \\
MC (kg) & $61,5 \pm 20,0$ & $55,8 \pm 14,9$ & $67,0 \pm 22,8$ & $\mathbf{0 , 0 4 0}$ \\
Estatura (cm) & $148,3 \pm 13,8$ & $145,5 \pm 12,6$ & $151,0 \pm 14,6$ & 0,145 \\
IMC $\left(\mathrm{kg} / \mathrm{m}^{2}\right)$ & $27,3 \pm 5,6$ & $25,9 \pm 3,9$ & $28,8 \pm 6,7$ & 0,062 \\
\%G & $32,1 \pm 6,2$ & $32,8 \pm 5,5$ & $31,4 \pm 6,9$ & 0,450 \\
CC $(\mathrm{cm})$ & $86,9 \pm 11,9$ & $82,2 \pm 9,7$ & $91,4 \pm 12,1$ & $\mathbf{0 , 0 0 4}$ \\
CT $(\mathrm{mg} / \mathrm{dL})$ & $151,6 \pm 27,1$ & $152,4 \pm 28,5$ & $150,9 \pm 26,3$ & 0,839 \\
HDL-C $(\mathrm{mg} / \mathrm{dL})$ & $43,6 \pm 6,0$ & $43,5 \pm 6,7$ & $43,7 \pm 5,3$ & 0,921 \\
LDL-C (mg/dL) & $88,4 \pm 26,4$ & $90,0 \pm 28,0$ & $86,8 \pm 25,3$ & 0,662 \\
TG (mg/dL) & $103,5 \pm 44,3$ & $104,3 \pm 44,6$ & $102,7 \pm 44,9$ & 0,897 \\
Glicemia (mg/dL) & $88,4 \pm 9,2$ & $81,9 \pm 8,5$ & $84,9 \pm 9,7$ & 0,231 \\
HOMA-RI & $2,9 \pm 2,8$ & $3,2 \pm 3,6$ & $2,7 \pm 1,8$ & 0,544 \\
\hline
\end{tabular}

MC: massa corporal; IMC: índice de massa corporal; \%G: percentual de gordura; CC: circunferência da cintura.

* Teste $t$ para amostras independentes $p<0,05$.
A Tabela 2 apresenta a classificação dos participantes de acordo com o com o \%G (moderadamente alto e alto). Observou-se diferença estatisticamente significante, além do próprio \%G, para o CT e LDL-C favorecendo os meninos e meninas com maior quantidade de tecido adiposo.

Na Tabela 3, são apresentadas a frequência absoluta (n) e relativa (\%) segundo pontos de corte para a classificação de risco cardiovascular das crianças e adolescentes com sobrepeso e obesidade de acordo com variáveis antropométricas, laboratoriais e de composição corporal. Observa-se que $50 \%$ ou mais das crianças e adolescentes apresentaram valores alterados para IMC, \%G, CC e HDL-C. Adicionalmente, embora as lipoproteínas CT e TG e a RI estejam com valores aumentados em uma parcela menor na amostra, a quantidade de crianças e adolescentes nestas condições revela uma preocupação maior, com percentuais de risco próximos a 45,3, 41,5 e 49,1\%, respectivamente.

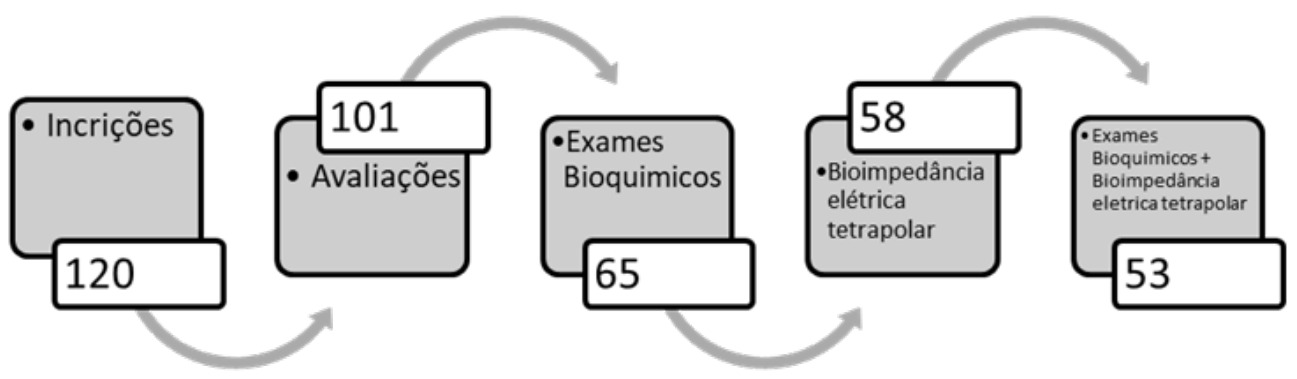

Figura 1. Fluxograma dos participantes.

Tabela 2. Distribuição dos fatores de risco cardiovasculares de acordo com a classificação do percentual de gordura corporal em crianças e adolescentes com sobrepeso e obesidade.

\begin{tabular}{lccc}
\hline Variáveis & $\begin{array}{c}\text { \%G } \\
\text { Moderadamente } \\
\text { alto }(\mathbf{n = 1 2})\end{array}$ & $\begin{array}{c}\text { \%G Alto } \\
(\mathbf{n = 4 1 )}\end{array}$ & $\boldsymbol{p}$ \\
\hline Idade (anos) & $11,7 \pm 2,7$ & $10,9 \pm 2,6$ & 0,342 \\
MC $(\mathrm{kg})$ & $57,3 \pm 19,0$ & $62,7 \pm 20,3$ & 0,414 \\
Estatura $(\mathrm{cm})$ & $150,2 \pm 15,6$ & $147,8 \pm 13,4$ & 0,591 \\
IMC $\left(\mathrm{kg} / \mathrm{m}^{2}\right)$ & $24,6 \pm 3,9$ & $28,1 \pm 5,9$ & 0,056 \\
\%G & $23,6 \pm 4,5$ & $34,6 \pm 4,1$ & $\mathbf{0 , 0 0 1}$ \\
CC (cm) & $83,5 \pm 10,5$ & $94,1 \pm 12,0$ & 0,052 \\
CT (mg/dL) & $134,2 \pm 21,5$ & $156,7 \pm 26,7$ & $\mathbf{0 , 0 1 0}$ \\
HDL-C (mg/dL) & $45,6 \pm 5,9$ & $43,0 \pm 5,9$ & 0,199 \\
LDL-C (mg/dL) & $74,9 \pm 28,0$ & $92,3 \pm 24,9$ & $\mathbf{0 , 0 4 4}$ \\
TC (mg/dL) & $82,7 \pm 41,7$ & $109,6 \pm 43,7$ & 0,064 \\
Glicemia (mg/dL) & $81,8 \pm 7,2$ & $83,9 \pm 9,7$ & 0,475 \\
HOMA-RI & $2,2 \pm 1,4$ & $3,2 \pm 3,1$ & 0,276 \\
\hline
\end{tabular}

\%G moderadamente alto: entre 20 a 25\% meninos e 25 a 30\% meninas; \%G Alto: maior 25 meninos e maior $30 \%$ meninas; CC - Circunferência da cintura. $p<0,05$; teste $t$ de Student.
Tabela 3. Classificação do risco cardiovascular de acordo com as variáveis antropométricas, laboratoriais e de composição corporal em crianças e adolescentes com sobrepeso e obesidade.

\begin{tabular}{lcc}
\hline Variáveis & Classificação & Frequência \% (n) \\
\hline IMC/idade (z-score) & Sobrepeso & $18,9(10)$ \\
& Obesidade & $60,4(32)$ \\
\%G & Obesidade Severa & $20,8(11)$ \\
CC (cm) & Moderado alto & $22,6(12)$ \\
& Alto & $\mathbf{7 7 , 5}(\mathbf{4 1})$ \\
CT (mg/dL) & P50 (normal) & $35,8(19)$ \\
& P90 (alterado) & $\mathbf{6 4 , 2}(\mathbf{3 4})$ \\
HDL-C (mg/dL) & Normal & $54,7(29)$ \\
& Alterado & $45,3(24)$ \\
LDL-C (mg/dL) & Normal & $32,1(17)$ \\
& Alterado & $\mathbf{6 7 , 9}(\mathbf{3 6})$ \\
TC (mg/dL) & Normal & $75,5(40)$ \\
Glicemia (mg/dL) & Alterado & $24,5(13)$ \\
HOMA-RI & Normal & $58,5(31)$ \\
& Alterado & $41,5(22)$ \\
\hline
\end{tabular}

MC: massa corporal; IMC: índice de massa corporal; \%G: percentual de gordura CC: circunferência da cintura; P50 e P90: pecentis 50 e 90. 


\section{DISCUSSÃO}

Os principais achados no presente estudo revelaram que as meninas, para a mesma idade, apresentam maior CC e MC do que os meninos, enquanto aos participantes com $\% \mathrm{G}$ alto (maior $25 \%$ meninos e $30 \%$ meninas) demonstraram níveis de CT e LDL-C superiores a valores de gordura corporal moderadamente alto (entre $20-25 \%$ meninos e 25 $30 \%$ meninas). Adicionalmente, mais de $50 \%$ dos meninos e meninas com sobrepeso e obesidade investigados, demonstram valores alterados para \%G, CC e HDL-C, e, uma parcela discretamente menor com valores alterados para CT, TG e RI.

Estudos com este perfil são relevantes, uma vez que houve um aumento significativo de crianças e adolescentes com excesso de peso nas últimas décadas ${ }^{3,5}$ sugerindo um risco cardiovascular cada vez maior nessa população, ${ }^{8}$. Durante muito tempo, persistiu a ideia de que DCV surgiam apenas em pessoas com idade avançada, porém, na atualidade, há suficiente evidência que o processo da aterosclerose iniciase em idade precoce ${ }^{7,8,17}$. O sobrepeso e a obesidade em crianças e adolescentes têm sido fortemente associado à vários fatores de risco cardiovascular ${ }^{7,8}$. Em estudo clássico, Gerber \& Zielinsky ${ }^{18}$, revelaram que os fatores de risco para aterosclerose estão presentes na infância e enfatizam que devem ser investigados.

Existe uma nítida relação entre aumento do IMC e prevalência dos fatores de risco cardiovascular ${ }^{17}$. Em estudo realizado com crianças e adolescentes de ambos os sexos com excesso de peso (8 a 16 anos) demonstrou que metade dos sujeitos apresentavam pelo menos um fator de risco biológico para as DCV ${ }^{19}$. Silva et al. ${ }^{7}$ apontam que, apesar de um único fator de risco contribuir por si só, para o desenvolvimento das DCV, os fatores de risco biológicos tendem a agregar-se, potencializando o aparecimento precoce das DCV. Essas informações sugerem que as crianças e adolescentes investigados, encontram-se em risco elevado, uma vez que foram observados alterações em fatores de risco fortemente associados a DCV como gordura total (\%) e abdominal (CC), CT e LDL-C.

Proporção elevada (73\%) de crianças a adolescentes canadenses com sobrepeso e obesidade apresentaram níveis lipídicos alterados e fortemente relacionados a composição corporal, especialmente abdominal ${ }^{20}$. Em outro estudo, uma parcela importante de crianças com sobrepeso e obesidade demonstraram níveis alterados para TG $(42,4 \%)$, LDL-C $(20,3 \%)$ e HDL-C $(6,8 \%)^{21}$. Há evidência sugerindo que quanto maior o excesso de peso na juventude, mais grave serão os distúrbios no metabolismo lipídico ${ }^{22}$. Essas informações corroboram com os resultados do presente estudo.
A obesidade infantil também está fortemente associada à resistência à insulina (RI) e alterações no metabolismo da glicose ${ }^{23}$. Em nosso estudo não houve diferenças entre meninos e meninas para os níveis glicêmicos e RI nem mesmo para diferentes níveis de gordura relativa (\%). Contudo, $49,1 \%(n=26)$ das crianças e adolescentes investigados, apresentaram valores de HOMA-RI superior a 2,5 unidades. No estudo de Sena et al. ${ }^{24}$ a RI foi confirmada em 36,8\% de crianças e adolescentes com excesso de peso. Entender a obesidade como um processo inflamatório é primordial para a compreensão da possibilidade do desenvolvimento da resistência à insulina precocemente. A resistência à insulina somada ao excesso de peso pode ser um importante gatilho para o aumento do processo inflamatório e alterações no metabolismo dos carboidratos e lipídeos ${ }^{25}$.

A CC é considerada um excelente indicador de adiposidade abdominal e está fortemente relacionada com as doenças cardiovasculares. Estudos observam que a presença da adiposidade central tem forte correlação com o desenvolvimento de dislipidemias e RI em idade pediátrica ${ }^{26}$. Os resultados de uma análise de coorte de adolescentes indicaram que a CC em crianças e adolescentes é extremamente útil na detecção de pacientes com síndrome metabólica e risco cardiovascular ${ }^{27}$. Em nosso estudo, a CC apresentou valores acima do P90 em 64,2\% da amostra. Crianças de 3 a 11 anos com CC acima do P90 (19\%) apresentaram maior número de fatores de risco para DCV do que crianças com valores abaixo do percentil $90(9,4 \%)^{28}$.

No presente estudo, foi verificado que as meninas são mais pesadas do que os meninos, para a mesma faixa etária, corroborando com outras pesquisas ${ }^{29,30}$. Em relação as alterações metabólicas, não há um consenso sobre a influência do sexo, uma vez que adolescentes crescem e amadurecem em diferentes taxas ${ }^{31,32}$. Hirscheler et al. ${ }^{33}$ mostraram que embora em sua amostra meninas e meninos apresentaram IMC e CC similar, as meninas foram mais resistente à insulina e tinham níveis mais adversos de lipídios do que meninos. Essa relação pode ser explicada pelo fato das meninas entrarem na puberdade mais cedo, e que o excesso de peso é uma condição que acaba adiantando mais o início dessa fase $\mathrm{e}^{34,35}$.

A quantidade e o local de deposição de gordura no corpo são fatores importantes das consequências da obesidade para a saúde. O padrão de gordura corporal parece estar fortemente associado com alterações no perfil lipídico em crianças e adolescentes com idades entre 6 a 18 anos, independente dos níveis de obesidade ${ }^{34,36}$. Em nosso estudo a média do percentual de gordura corporal foi de 32,1\% (Tabela 1) e $77,5 \%$ (Tabela 3) dos meninos e meninas 
demonstram valores elevados de gordura corporal. Estudos apontam que crianças e adolescentes com alta quantidade de gordura corporal (maior 25\% meninos e 30\% meninas) apresentam elevado risco de desenvolvimento de $\mathrm{DCV}^{37}$.

Os fatores de risco para DCV investigados no presente estudo, não permitem a identificação de relação de causalidade com o sexo e o \%G, uma vez que o delineamento foi transversal. No entanto, os dados são indicadores úteis para a atuação clínica e podem ajudar a orientar políticas de saúde, focalizando na reversão do quadro de crianças e adolescentes com excesso de peso.

Conclui-se que a prevalência de fatores de risco para doenças cardiovasculares é elevada neste grupo de crianças e adolescente com sobrepeso e obesidade. As meninas demonstraram maior CC e MC do que os meninos e, quanto maior o \%G, maiores são os níveis de CT e LDL-C. Ainda, mais da metade dos meninos e meninas demonstram valores alterados para \%G, CC e HDL-C, e, uma parcela discretamente menor com valores alterados para CT, TG e RI. Considerando que as DCV se iniciam na infância, progridem com a idade e exibem gravidade diretamente proporcional ao número de fatores de risco apresentados pelo indivíduo, acreditamos na relevância da prevenção primária nesta faixa etária com ênfase na dieta e na manutenção de uma prática regular de atividade física para toda a vida.

\section{AGRADECIMENTOS}

Secretaria de Estado da Ciência, Tecnologia e Ensino Superior (SETI) do Paraná. Programa de Extensão Universidade sem Fronteiras (USF), Subprograma Apoio à Saúde (Edital 02/2011 e convenio 6/2012/SETI/UGF).

\section{REFERÊNCIAS}

1. Duncan BB, Chor D, Aquino EML, Bensenor IM, Mill JG, Schmidt MI, Lotufo PA, Vigo A, Barreto SM. Doenças crônicas não transmissíveis no Brasil: prioridade para enfrentamento e investigação. Rev Saúde Públ. 2012;46(1Suppl):126-34. https://doi.org/10.1590/S003489102012000700017

2. Silva VS, Petroski EL, Souza GI, Silva DAS. Prevalência e fatores associados ao excesso de peso em adultos do Brasil: um estudo de base populacional em todo território nacional. Rev Bras Ciênc Esporte. 2012;34(3):713-26. https://doi.org/10.1590/S010132892012000300013

3. Ministério da Saúde (Brasil), Secretaria de Vigilância em Saúde, Departamento de Análise de Situação de Saúde. Vigilância de Fatores de Risco e Proteção para Doenças Crônicas por Inquérito Telefônico, Vigitel 2009. Brasília: Ministério da Saúde; 2010.

4. Instituto Brasileiro de Geografia e Estatística (IBGE). POF 20082009 - Antropometria e estado nutricional de crianças, adolescentes e adultos no Brasil. IBGE; 2010.
5. Fraporti MI, Adami FS, Rosolen MD. Fatores de risco cardiovascular em crianças. Rev Port Cardiol. 2016;36(10):699-705. https://doi. org/10.1016/j.repc.2016.12.013

6. Araújo J, Ramos E. Paediatric obesity and cardiovascular risk factors - A life course approach. Porto Biomed J. 2017;2(4):102-10. https:// doi.org/10.1016/j.pbj.2017.02.004

7. Silva LR, Stefanello JMF, Pizzi J, Timossi LS, Leite N. Aterosclerose subclínica e marcadores inflamatórios em crianças e adolescentes obesos e não obesos. Rev Bras Epidemiol. 2012;15(4):804-16. https://doi.org/10.1590/S1415-790X2012000400012

8. Barbalho SM, Oshiiwa M, Fontana LCS, Finalli EFR, Paiva Filho ME, Spada APM. Metabolic syndrome and atherogenic indices in school children: A worrying panorama in Brazil. Diabetes Metab Syndr. 2017;11 Suppl 1:S397-S401. https://doi.org/10.1016/j. dsx.2017.03.024

9. Lohman TG, Roche AF, Martorel R. Anthropometric standardization reference manual. Champaign (IL): Human Kinetics Books; 1988.

10. Onis M, Onyango AW, Borghi E, Siyam A, Nishida C, Siekmann J. Development of a $\mathrm{WHO}$ growth reference for school-aged children and adolescents. Bull World Health Organ. 2007;85(9):660-7. https://doi.org/10.2471/BLT.07.043497

11. Freedman DS, Serdula MK, Srinivasan SR, Berenson GS. Relation of circumferences and skinfold thicknesses to lipid and insulin concentrations in children and adolescents: the Bogalusa Heart Study. Am J Clin Nutr. 1999;69:308-17. https://doi.org/10.1093/ ajcn/69.2.308

12. Lohman TG. Assesing fat distribuition. In: Lohman TG, editor. Advances in body composition assessment: current issues in exercise science. Champaign, IL: HumanKinetics; 1992. p. 57-63.

13. Friedewald WT, Levy RI, Fredrickson DS. Estimation of the concentration no flow density lipoproteins cholesterol in plasma without use of the ultracentrifuge. Clin Chem. 1972;18(6):499-502.

14. Sociedade Brasileira de Cardiologia. I Diretriz de Prevenção da Aterosclerose na Infância e na Adolescência. Arq Bras Cardiol. 2005;85(6):3-36.

15. Matthews DR, Hosker JP, Rudenski AS, Naylor BA, Treacher DF, Turner RC. Homeostasis model assessment, insulin resistance and $\beta$-cell function from fasting plasma glucose and insulin concentration in man. Diabetologia. 1985;28(7):412-9. https://doi.org/10.1007/ BF00280883

16. d'Annunzio G, Vanelli M, Meschi F, Pistorio A, Caso M, Pongigline $C$, et al. The SIDEP Study Group. Valori normali di HOMA-IR in bambini e adolescenti: studio multicentrico italaiano. Quad Pediatr. 2004;3:44.

17. Garcés C, Oya M. Factores de riesgo cardiovascular en la edad infantil. Resultados globales del estudio Cuatro Provincias. Rev Esp Cardiol. 2007;60(4):517-24. https://doi.org/10.1016/S03008932(07)75069-8

18. Gerber ZRS, Zielinsky P. Fatores de Risco de Aterosclerose na Infância. Um Estudo Epidemiológico. Arq Bras Cardiol. 1997:69(4):231-36. https://doi.org/10.1590/S0066-782X1997001000002

19. Andersen LB, Harro M, Sardinha LB, Froberg K, Ekelund U, Brage $S$, Anderssen AS. Physical activity and clustered cardiovascular risk in children: a cross-sectional study (The European Youth Heart Study). Lancet. 2006:368(9532):299-304. https://doi.org/10.1016/S01406736(06)69075-2 
20. Rueda-Clausen C, Ambler KA, Ball GDC, Sharma AM, Padwal RS. Lipid profile abnormalities among children and adolescents with severe obesity. Can J Diabetes. 2013;37:S239. https://doi. org/10.1016/j.jcjd.2013.03.140

21. Buff CG, Ramos E, Souza FIS, Sarni ROS. Frequência de síndrome metabólica em crianças e adolescentes com sobrepeso e obesidade. Rev Paul Pediatr. 2007;25(3):221-6. https://doi.org/10.1590/S010305822007000300005

22. Fujii C, Sakakibara H. Association between insulin resistance, cardiovascular risk factors and overweight in Japanese schoolchildren. Obes Res Clin Pract. 2012;6(1):e1-e90. https://doi.org/10.1016/j. orcp.2011.04.002

23. Bezerra AC, Sampaio HAC, Melo MLP, Maia SCS, Almeida PC. Associação entre dislipidemia e excesso de peso de crianças e adolescentes atendidos em uma unidade de saúde. Rev. Baiana Saúde Pública. 2011;35(2):348-62.

24. Sena ALS, Cardoso AD, Carvalho DFC, Medeiros JL, Coutinho GF, Albuquerque FCL, Medeiros, CCM. Excessive daytime sleepnessand cardiometabolic risk factors in children and teenagers with overweight. JHGD. 2013;23(1):24-30. https://doi.org/10.7322/ jhgd.50387

25. Uysal KT, Wiesbrock SM, Marino MW, Hotamisligil GS. Protection from obesity-induced insulin resistance in mice lacking TNG-alpha function. Nature. 1997;395(6651):610-4.

26. Bergmann GG, Gaya A, Halpern R, Bergmann MLA, Rech RR, Constanzi CB, Alli LR. Waist circumference as screening instrument for cardiovascular disease risk factors in schoolchildren. J Pediatr. 2010;86(5):411-6. https://doi.org/10.2223/JPED.2026

27. Yamborisut U, Sakamoto N, Wimonpeerapattana W, Tontisirin K. Waist circumference and body fat distribution indexes as screening tools for the overweight and obesity in Thai preschool children. Obes Res Clin Pract. 2010;4(4):307-14. https://doi.org/10.1016/j. orcp.2010.08.001

28. Reinehr T, Wunsch R. Relationships between cardiovascular risk profile, ultrasonographic measurement of intra-abdominal adipose tissue, and waist circumference in obese children. Clin Nutr. 2010;29(1):24-30. https://doi.org/10.1016/j.clnu.2009. 06.004
29. Ramírez-Vélez R, García-Hermoso A, Agostinis-Sobrinho C, Mota J, Santos R, Correa-Bautista JE, Amaya-Tambo DC, Villa-González E. Cycling to school and body composition, physical fitness, and metabolic syndrome in children and adolescents. J Pediatr. 2017;188:57-63. https://doi.org/10.1016/j.jpeds.2017.05.065

30. Spolidoro JV, Filho MLP, Vargas LT, Santana JC, Pitrez E, Hauschild JA, Bruscato NM, Moriguchi EH, Medeiros AK, Piva JP. Waist circumference in children and adolescents correlate with metabolic syndrome and fat deposits in young adults. Clin Nutr. 2013;32(1):937. https://doi.org/10.1016/j.clnu.2012.05.020

31. Peper JS, Brouwer RM, Schnack HG, van Baal GC, van Leeuwen M, van den Berg SM, Delemarre-Van de Waal HA, Boomsma DI, Kahn RS, Hulshoff Pol HE. Sex steroids and brain structure in pubertal boys and girls. Psychoneuroendocrinology. 2009;34(3):332-42. https://doi.org/10.1016/j.psyneuen.2008.09.012

32. Maffeis C, Pietrobelli A, Grezzani A, Provera S, Tato L. Waist circumference and cardiovascular risk factors in prepubertal children. Obes Res. 2001;9(3):179-87. https://doi.org/10.1038/ oby. 2001.19

33. Hirscheler V, Maccallini G, Karam C, Gonzalez C, Aranda C. Are girls more insulin-resistant than boys? Clin Biochem. 2009;42(1011):1051-6. https://doi.org/10.1016/j.clinbiochem.2009.03.002

34. Bonaccorsi G, Baggiani L, Bassetti A, Colombo C, C, Mantero S, Olimpi N, Santomauro F, Comodo S. Body composition assessment in a sample of eight-year-old children. Nutrition. 2009;25(10):10208. https://doi.org/10.1016/j.nut.2009.01.016

35. Doak CM, Hoffman DJ, Norris SA, Ponce MC, Polman K, Griffiths PL. Is body mass index an appropriate proxy for body fat in children? Glob Food Sec. 2013;2(2):65-71. https://doi.org/10.1016/j. gfs.2013.02.003

36. Freedman DS, Ogden CL, Berenson GS, Horlick M. Body mass index and body fatness in childhood. Curr Opin Clin Nutr Metab Care. 2005;8(6):618-23. https://doi.org/10.1097/01. mco.0000171128.21655.93

37. Ma J, Feng N, Zhang S, Pan Y, Huang Y. Comparison of Changes in Body Composition during Puberty Development of Obese and Normal-weight Children in China. Biomed Environ Sci. 2009; 22(5):413-8. https://doi.org/10.1016/S0895-3988(10)60019-0 